
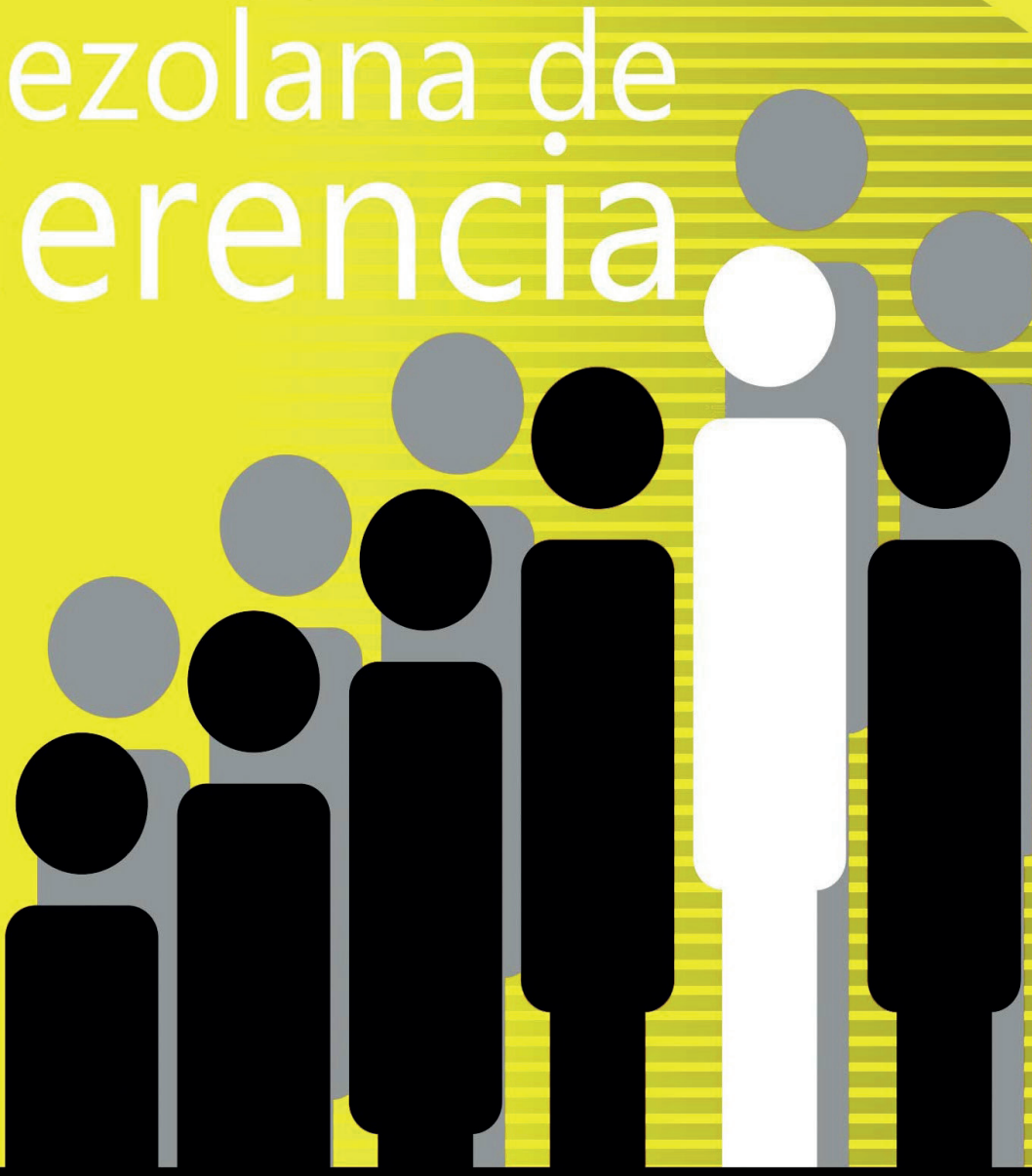


\title{
Gestión de personas y las barreras para innovar en la transformación digital ${ }^{*}$
}

\author{
Maliqueo Pérez, Carolina ${ }^{* *}$ \\ González Candia, Julio** \\ Mardones Espinosa, Regina* \\ Ardiles Briones, Mauricio
}

\section{Resumen}

El artículo expone los principales resultados de un estudio cuyo objetivo fue explicar de manera integrada las principales barreras que se presentan en el área de gestión de personas en empresas chilenas para incorporar la innovación y la transformación digital. En términos metodológicos, se utilizó un enfoque cuantitativo en un nivel, preferentemente, descriptivo. Se aplicó una encuesta a 91 representantes de empresas utilizando un criterio muestreal intencionado. En los resultados se identificaron las principales barreras del área de gestión de personas para innovar en la transformación digital, las acciones de innovación más frecuentes, el tipo de tecnología utilizada en los procesos, el grado de conocimientos que poseen las y los profesionales del área y el nivel de posicionamiento estratégico de la unidad en estos ámbitos. Se concluye que, se necesita un nuevo enfoque estratégico de la visión de la gerencia general hacia la gestión de personas y un cambio en sus funciones, de sus procesos y servicios y que utilice la innovación y la digitalización como factores de éxito para la transformación digital.

Palabras clave: Gestión de Personas; Barreras; Innovación; Transformación Digital.

\section{Recibido: 21.09.20 Aceptado: 15.02.21}

A la Srta. María Raquel González y al Sr. Boris Riveros Valdés por su trabajo y colaboración en los ajustes finales de formato del presente artículo. Al Depto. de Tecnologías de Gestión de la Facultad Tecnológica de la Universidad de Santiago de Chile por contribuir en que este artículo se pudiera concretar durante el presente año 2021.

* Licenciada en Organización y Gestión Tecnológica y Tecnóloga en Administración de Personal, Magíster en Gestión de la Innovación y el Emprendimiento Tecnológico, Directora Observatorio de Recursos Humanos ORH Chile. carolina.maliqueo.p@gmail.com ORCID: 0000-0002-3308-6432

*** Administrador de Personal y de Industrias. Magíster en Educación. Doctor en Procesos Sociales y Políticos en América Latina. Departamento de Tecnologías de Gestión, Facultad Tecnológica, Universidad de Santiago de Chile. julio.gonzalez@usach.cl ORCID: 0000-0003-4629-0982

**** Psicóloga, Lic. en Psicología. Master en Dirección Estratégica de Recursos Humanos. Departamento de Tecnologías de Gestión, Facultad Tecnológica, Universidad de Santiago de Chile. Correo electrónico: regina. mardones@usach.cl ORCID: 0000-0002-3329-6376

***** Psicólogo, Lic. en psicología. Magíster en Gestión de la Innovación y el Emprendimiento Tecnológico. Docente Departamento de Tecnologías de Gestión, Facultad Tecnológica. Correo electrónico: mauricio.ardiles@ usach.cl ORCID: 0000-0002-7089-6696 


\title{
People management and barriers to innovation in digital transformation
}

\begin{abstract}
The article presents the main results of a study whose objective was to explain in an integrated way the main barriers that arise in the area of people management in Chilean companies to incorporate innovation and digital transformation. In methodological terms, a quantitative approach was used at a level, preferably descriptive. A survey was administrated to 91 company representatives using an intentional sampling criterion. The results identified the main barriers in the area of people management to innovate in the digital transformation, the most frequent innovation actions, the type of technology used in the processes, the degree of knowledge that the professionals in the area possess and the level of strategic positioning of the unit in these areas. It concludes that a new strategic approach is needed to the general management's vision towards people management and a change in their functions, processes and services, and to use innovation and digitalization as success factors for digital transformation.
\end{abstract}

Keywords: People Management, Barriers, Innovation, Digital Transformation.

\section{Introducción}

Hablar hoy de innovación es habitual en el ámbito empresarial, para Herrera e Hidalgo (2019) y Vilaplana y Stein (2020) es considerada como uno de los atributos más valiosos de la economía digital y que contribuye con más valor para la sostenibilidad de las organizaciones. La irrupción de las tecnologías de la información y digitales han acortado los ciclos de vida de los productos y los modelos de negocio son mucho más dinámicos. En este contexto y según Eguren (2019), el presente trabajo se justifica en la necesidad de desarrollar un estudio contextualizado a la realidad chilena, dado que no se ha apreciado en este ámbito una investigación que se enfocara en las barreras u obstáculos del área de gestión de personas o recursos humanos para innovar en su modelo de gestión, incorporando de manera estratégica elementos de la transformación digital. De esta forma, el objetivo principal del trabajo fue explicar de manera integrada las principales barreras que se presentan en las áreas de gestión de personas en empresas chilenas para incorporar la innovación y la transformación digital en su modelo de gestión y transformarse en un agente de cambio para la organización.

Para estos efectos y en la perspectiva de Baena (2017), se formularon las siguientes preguntas centrales de la investigación: ¿qué están haciendo en Chile las áreas de recursos humanos en su rol para adaptarse a los nuevos desafíos de las empresas en materia de transformación digital?, ¿cuáles son las herramientas 
Maliqueo Pérez, Carolina; González Candia, Julio; Mardones Espinosa, Regina; Ardiles Briones, Mauricio

Gestión de personas y las barreras para innovar en la transformación digital

que debiesen incorporar las áreas de Recursos Humanos para responder a las necesidades de las y los trabajadores y de la organización en la era digital? y ¿qué elementos están obstaculizando la innovación y la transformación digital dentro del área?.

Respecto de la metodología, se utilizó un enfoque cuantitativo en un nivel, preferentemente, descriptivo. Se diseñó, validó y aplicó una encuesta a 91 representantes de empresas utilizando un criterio muestreal intencionado. Las principales limitantes del estudio estuvieron dadas por la confidencialidad de los datos de las empresas, la temática o los tópicos abordados que en ciertos casos impidió que las encuestas se completaran adecuadamente y el haber trabajado con una muestra del tipo no probabilística, la cual no permite extrapolar los resultados obtenidos a grupos más amplios.

\section{Innovación, Transformación Digital y Gestión de Personas}

La Organización para la Cooperación y el Desarrollo Económico (OCDE - 2018) ${ }^{1}$ y Jiménez y Geldes (2019) reconocen la contribución de la innovación como factor clave para el desarrollo económico de los países y de las organizaciones. Según la OCDE, la digitalización se presenta como un factor que transforma los procesos de innovación y la promueve en los ámbitos de colaboración y abierta. El impacto de las tecnologías digitales avanza más rápido que las propias innovaciones dentro de las empresas, la capacidad de adaptación para dar respuestas a las nuevas demandas de los clientes, sean internos o externos es fundamental para la propia adaptación de la empresa con su entorno (OECD/Eurostat, 2018).

Se vive en una sociedad de la información, con distintas demandas y de distinto nivel de complejidad. Por tanto, es necesario que se desarrolle la creatividad, para que exista total coordinación con mayor sinergia en las acciones y estas sean eficaces $y$ decisivas para afrontar los diversos problemas complejos al interior de las organizaciones (Torres y Vargas, 2020:49). Transformar los modelos de negocio clásicos a digitales requiere innovación y cambios medulares en los procesos organizacionales y en la forma de trabajar de los colaboradores en el día a día.

El estudio de la IDC ${ }^{2}$ (2017), sugiere que Recursos Humanos tenga un papel fundamental en la transformación digital como agente de cambio desde dos ámbitos, asesor e implementador (Altimiras, 2017). Este mismo estudio reveló que en las empresas de alto crecimiento, las áreas de IT, de Negocios y de Recursos Humanos están muy alineadas, siendo fundamental que esta última apoye a superar las barreras que obstaculizan la transformación digital de las organizaciones. Estos son los factores claves donde recursos humanos podría innovar en su gestión y trabajar como socio estratégico (Ulrich, 2006), en línea con las gerencias de negocios y de C-suite ${ }^{3}$ (Revista Forbes,

3 Un argot usado para referirse colectivamente a los ejecutivos seniors más importantes de una corporación según Revista Forbes en 2017. 
2017) para el cambio, comprometiendo a los colaboradores en estos procesos de transformación. El Manual de Oslo de la OCDE (2018) establece la siguiente definición para la innovación; "es un producto o proceso nuevo o mejorado (o una combinación de los mismos) que difiere significativamente de los productos o procesos anteriores de la unidad y que ha sido puesto a disposición de usuarios potenciales (producto) o puesto en uso por la unidad (proceso)".

En comparación con la edición de 2005 del mismo manual, se realizó un cambio importante en la definición de innovación empresarial, la definición anterior incluía cuatro tipos de innovaciones: producto, proceso, organización y marketing, y la actual edición sólo propone dos tipos: innovaciones de productos e innovaciones de procesos de negocio. En este se define la innovación de un producto como "un bien o servicio nuevo o mejorado que difiere significativamente de los bienes o servicios anteriores de la empresa y que se ha introducido en el mercado" y la definición de innovación de un proceso de negocio o firma "como un proceso comercial nuevo o mejorado para uno o más funciones comerciales que difieren significativamente de los negocios anteriores de la empresa procesos y eso ha sido puesto en uso por la empresa" (OECD/Eurostat, 2018).

En Chile, uno de los instrumentos fundamentales para medir la innovación es la encuesta en empresas cuyo objetivo es entregar información sobre la estructura del proceso de innovación de las empresas (insumos y resultados) y mostrar las relaciones entre dicho proceso y la estrategia innovativa de las empresas, el esfuerzo innovativo, los factores que influyen en su capacidad para innovar y el rendimiento económico de las empresas. La encuesta mide las actividades que realizan las empresas chilenas de distintos sectores y regiones del país. Los resultados de la Onceava Encuesta Nacional de Innovación y Encuesta Nacional de I $+\mathrm{D}^{4}$, presentada ahora por el nuevo Ministerio de Ciencia, Tecnología, Conocimiento e Innovación nos presenta tres resultados a destacar: 1) El porcentaje de empresas que innovan (medición según enfoque de Eurostat), Chile presenta un $21,4 \%{ }^{5}$. 2) La tasa de innovación, la cual establece el porcentaje de empresas que realizaron algún tipo de innovación en Productos, Procesos, Organizacional o Marketing durante 2017-2018 fue de un $14.1 \%$. Esto representa una baja de 1 punto porcentual en comparación al último estudio realizado en 2015-2016, cuya tasa llegó a $15.1 \%$. Y 3) El total de empresas consideradas en la encuesta en este último periodo fue de 175.288.De este total, un $4,6 \%$ de las empresas "innovan en productos" y un $12,6 \%$ "innovan en procesos de negocios".

4 Encuesta Nacional de Innovación y Encuesta Nacional de I + D. Documento fue elaborado por la División de Estudios y Estadísticas de la Subsecretaría de Ciencia, Tecnología, Conocimiento e Innovación. En https:// www.minciencia.gob.cl/sites/default/files/metodologia encuestas eni e id 2.pdf fecha de consulta 10 de octubre de 2020.

5 A nivel mundial se consideran datos entre los años 2014 - 2016. Para Chile se imputa el dato de la Encuesta Nacional de Innovación, años de referencia 2017-2018.

6 Se introdujeron cambios importantes al cuestionario. Estos se aplicaron en la ENI 2017-2018 y se centraron en adecuar las preguntas a las nuevas definiciones de innovación de productos y procesos de negocios. El cambio más drástico es que esta última pasó a agrupar los antiguos tipos de innovación en procesos marketing y organizacional (Minciencia,2020:22) 

Briones, Mauricio

Gestión de personas y las barreras para innovar en la transformación digital

No deja de llamar la atención que nuevamente estamos frente a una baja porcentual en la tasa de innovación a nivel nacional. La baja se venía produciendo en las mediciones de los años 2015-2016 (15.1\%) respecto del periodo $2013-2014(16.6 \%)$ y de este último con relación al periodo 2011-2012 (23.7\%) (Minciencia, 2020:72 a 73). En este sentido, el auge tecnológico hasta la época actual, han ocurrido importantes cambios en el mundo, orientados a propover la actividad innovativa por resolver problemas, y a la experiencia acumulada dentro de quienes la desarrollan (Piña y Senior, 2020).

En lo que respecta a la transformación digital, Walker, et al, (2016) dice que la transformación empresarial digital es el proceso de explotación de tecnologías digitales y capacidades de soporte para crear un nuevo modelo de negocio digital robusto. Crespo y Pariente (2018) conciben la transformación digital como el proceso de gestión que orienta la cultura, la estrategia, las metodologías y las capacidades de una organización a partir de las tecnologías digitales. Power Data (2020), plantea que la transformación digital, es entendida como "la aplicación de capacidades digitales a procesos, productos y activos para mejorar la eficiencia, mejorar el valor para el cliente, gestionar el riesgo y descubrir nuevas oportunidades de generación de ingresos"7.
La mayoría de las empresas han utilizado herramientas digitales por mucho tiempo, operan computadoras para procesos y datos operativos importantes. Hablar de transformación digital según Cobo (2008) y Fitzgerald, et al, (2013), se relaciona con las posibilidades que otorgan las tecnologías digitales que se han extendido en forma masiva y comprender cómo usarlas para mejorar la productividad y el desempeño del negocio. Para que ello ocurra, las empresas y las áreas deben dominar tres factores. Primero la tecnología, el qué necesita la empresa y segundo como se conecta con su actual modelo de negocio.

Finalmente, se trata de la forma en como la empresa en forma ágil la utiliza para lograr los resultados (McKinsey, 2019). Se puede señalar que todas las definiciones apuntan a la incorporación de las tecnologías digitales como una herramienta para mejorar el desempeño del negocio ya sea mejorando su actual modelo o transformándolo en otro nuevo, con el cliente y las y los trabajadores en el centro de su propósito.

Según Ferrándiz (2017) y Maliqueo (2019) para las empresas, la transformación digital implica, oportunidad, competitividad, flexibilidad, crecimiento, innovación, liderazgo, personalización, atracción y desarrollo del talento, adaptación a las necesidades de los clientes y nuevos ingresos ${ }^{8}$. Es importante hacer otra distinción, no es

7 En https://www.powerdata.es/transformacion-digital visitada el 01 de mayo de 2020. En este mismo sitio web especializado se precisan las "capacidades digitales", dando a entender que estas son electrónicas, científicas, basadas en datos, cuantificadas, instrumentadas, medidas, calculadas y muy posiblemente automatizadas.

8 Para Ferrándiz (2017) la principal causa de la transformación digital en las organizaciones, ha sido el cambio en los hábitos de consumo de las personas producto del uso de tecnología digital, al alcance de las personas a través de dispositivos móviles capaces de transmitir y recibir datos en tiempo real. 
lo mismo digitalizarse que transformarse digitalmente, incorporar tecnología es digitalizarse, transformarse digitalmente es el uso que se le da a la tecnología para mejorar los productos, servicios o crear nuevos modelos de negocios para solucionar las necesidades de los clientes sean internos o externos.

La digitalización actúa como impulsor de un mejor desempeño de las organizaciones para abordar las necesidades de sus clientes y usuarios, al respecto Barros (2016) señala que la digitalización se está desplegando en tres etapas: 1) "Cliente Digital", 2) "Empresa Digital"10 y 3) "Operaciones Digitales"11; esta última correspondería a la etapa más disruptiva en términos de productividad e innovación.

La transformación digital según Khin y Ho (2019), es lo que permite a las empresas utilizar el impacto de las tecnologías digitales para crear nuevo valor, donde la digitalización no es un fin, sino es un acelerador de la transformación de las organizaciones que promueve la colaboración entre los empleados, es decir, aporta flexibilidad y agilidad entre las funciones corporativas (IT Digital, RR.HH., Finanzas) y las hace más flexibles y eficientes a las necesidades del negocio (Barros, 2016). La transformación no es solo digital, sino también cultural y organizacional. Para adaptarse las empresas deben transformarse en todos los ámbitos.
Esto ha permitido, en la perspectiva de Denning (2016), el surgimiento de nuevas formas de trabajo, más colaborativo, ágil y flexible, logrando que los equipos sean más eficientes, reduciendo en gran medida, el margen de error y los tiempos de espera ante las respuestas a clientes. Estos nuevos estilos de trabajo demandan de recientes habilidades digitales y tecnológicas.

Ahora bien, al hablar de la transformación digital y su impacto en la gestión de personas, es importante reconocer que la transformación digital y sus principales cambios, en los que resaltan las características de los clientes actuales, los nuevos modelos de negocios, y las nuevas formas de trabajar, demandan de las organizaciones adecuarse a un escenario empresarial y laboral diferente.

La transformación digital se inicia con una manera distinta de pensar que demanda un cambio importante en la cultura, la estructura y las operaciones de las organizaciones (Cuenca-Fontana, Matilla y Compte-Pujol, 2020). En la cuarta revolución industrial el trabajo está cambiando y este cambio está siendo mucho más profundo y de mayor alcance a lo imaginado (Schwab, 2016; Torrecilla, Pardo y Rubio, 2019). Las áreas de gestión y talento humano vienen evaluando nuevos perfiles y competencias para los cargos que tradicionalmente deben cubrir en las

9 Las empresas utilizan las tecnologías para ofrecer multicanal personalizada, generar mejores experiencias al usuario, sean clientes externos o los propios colaboradores.

10 Facultada por nuevos modelos operativos y procesos de negocio, permite utilizar las nuevas capacidades digitales y de automatización, para reducir costos para mejorar la eficiencia de las funciones corporativas.

11 Utilizando nuevas tecnologías como el "Internet de las cosas", la robótica, y la inteligencia artificial para automatizar las tareas rutinarias y las de mantenimiento. 
Maliqueo Pérez, Carolina; González Candia, Julio; Mardones Espinosa, Regina; Ardiles Briones, Mauricio

Gestión de personas y las barreras para innovar en la transformación digital

empresas, pero con la dificultad que los requisitos propios de los mismos han mutado de algunas competencias claramente medibles y evaluables, a otras denominadas blandas, donde prima el trabajo conjunto, los buenos resultados, la capacidad de asimilar el cambio, nuevas formas de remuneración y compensación de resultados, todo lo cual es producto de la era digital (Alexander, Stefanova y Zahidi, 2018).

A partir de los planteamientos ya revisados de la OCDE (2018), Altimiras (2017), Ulrich (2006), Crespo y Pariente (2018), Power Data (2020) y McKinsey (2019), se deduce que la gestión de personas tiene desafíos importantes por implementar en etapas sucesivas: 1 . Digitalizarse, optimizando los actuales procesos de recursos humanos para mejorar la atención y calidad de los servicios. Esto implica disminuir la labor transaccional operacional del área. 2. Transformarse digitalmente, utilizando los beneficios de las tecnologías digitales para la obtención de datos, transformándolos en conocimientos para innovar en nuevos y mejores servicios para las y los colaboradores y la organización. 3. Diseñar un nuevo modelo de gestión de personas basado en una propuesta de valor para las personas en función de las nuevas tendencias en esta materia, como son; ser una empresa saludable, flexible, diversa e inclusiva, temas que las nuevas generaciones de colaboradores valoran de las organizaciones. Y 4. Ser un verdadero agente de cambio, las áreas de gestión de personas se convierten en facilitadores de la transformación con capacidad para gestionar una nueva cultura digital, mejorar el desempeño, la productividad organizacional y asesorar a la Gerencia General en objetivos estratégicos coherentes a los nuevos escenarios laborales que se enfrentan (Ulrich, 1997) ${ }^{12}$.

Maliqueo y González (2020), destacan que los planteamientos propuestos por Dave Ulrich (1997) y Martínez y Bello (2017), se vinculan directamente con esta investigación explicando su teoría de los cuatros roles que existen en las áreas de gestión de personas dentro de las organizaciones, estos son; socio estratégico, agente de cambio, experto en administración y gestión y, líder de efectividad y socio de trabajadores. Por otra parte, presenta a su vez cuatro enfoques: orientado a procesos, a las personas, un enfoque estratégico o a futuro $y$, el enfoque operativo, que es del trabajo diario. Estos planteamientos resultan útiles para el presente estudio dado que son las categorías teóricas contra las cuales vamos a comparar los resultados de la aplicación de la encuesta y que se apreciaran más adelante, especialmente en cuanto a los roles que predominan en el actuar organizacional de las y los informantes.

El detalle de las principales responsabilidades en cada enfoque se puede apreciar en el diagrama 1:

12 Martínez y Bello (2017) plantean que para trabajar el proceso de digitalización internamente es necesario apoyarse en la función de personas, de la misma manera que durante el proceso de digitalización de cliente se busca el apoyo del Chief Marketing Officer. 
pp. $510-532$

Revista Venezolana de Gerencia, Año 26 No. 94, 2021

\section{Diagrama 1 \\ Modelo de 4 roles y 16 responsabilidades de Ulrich}

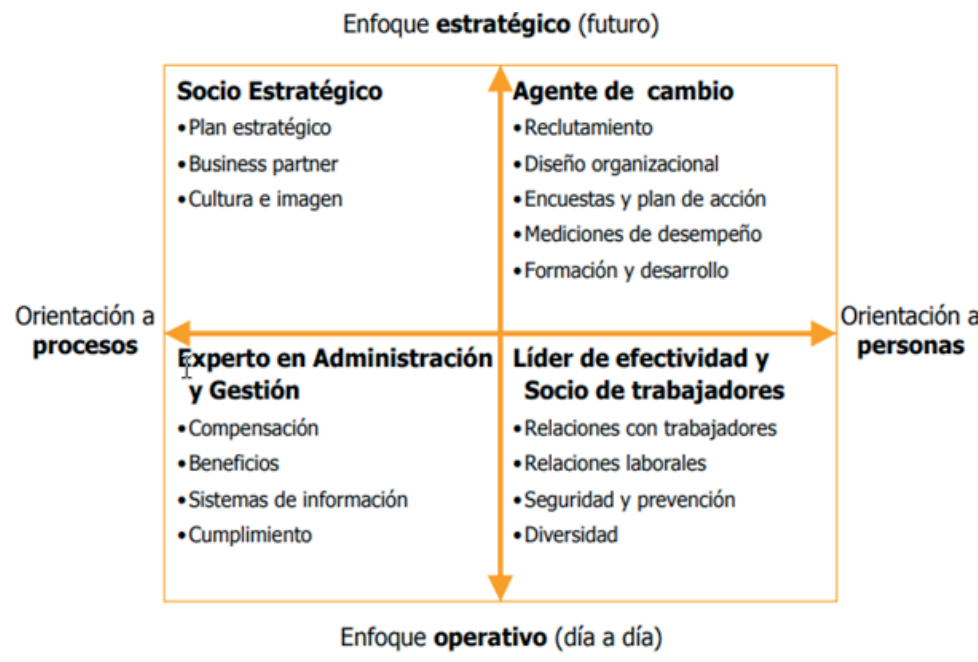

Fuente: Uzkudun (2014).

Este modelo, adicionalmente, permitió identificar donde se concentra la mayor dedicación de las áreas de gestión de personas en las empresas consultadas, lo que sirvió también como un indicador del nivel de madurez en innovación y tecnología.

\section{Consideraciones metodológicas}

El estudio tuvo un enfoque del tipo cuantitativo (Hernández y Mendoza, 2018) y el nivel de este fue preferentemente del tipo descriptivo (Reyes y Boente, 2019). De manera consistente con el enfoque adoptado, se diseñó, validó y aplicó un instrumento tipo encuesta para la recopilación de datos en una muestra seleccionada de profesionales. El diseño metodológico de este estudio fue no experimental del tipo transeccional o transversal, dado que los datos se recogieron en un momento único.

Las principales acciones metodológicas realizadas son las que a continuación se indican: Etapa I: Definición de las variables del estudio: se seleccionaron y definieron operacionalmente las principales variables que se iban a medir en la encuesta ${ }^{13}$. Etapa II: Diseño y validación de la Encuesta: se diseñó

13 Las principales variables independientes que se consideraron fueron: Tamaño de la Empresa (medianas y grandes), Nivel de Innovación, Transformación Digital en la empresa y Nivel de conocimientos y capacitación de las y los profesionales de las áreas de Recursos humanos. La principal variable dependiente fue: Nivel de incorporación de la innovación y la transformación digital en la gestión de las empresas en Chile 

Briones, Mauricio

Gestión de personas y las barreras para innovar en la transformación digital

inicialmente la encuesta para la recopilación de datos en base a diversas investigaciones realizadas con este tipo de metodología ${ }^{14}$. La encuesta diseñada fue validada por nueve (09) expertos y expertas disciplinarias/sectoriales y del mundo académico durante el mes de agosto de 2019. Para validar el contenido especializado en tecnología se solicitó también la revisión de un experto consultor en tecnología, con amplia y destacada experiencia internacional en la implementación de software ERP's de clase mundial. Y Etapa III: Prueba Piloto de la encuesta: con fecha 29 de agosto 2019 se realizó una validación piloto de la encuesta a cinco (5) ejecutivos que trabajaban en áreas de gestión de personas y, que contaban con más de veinte años de experiencia laboral y que fueron seleccionados por sus años de ejercicio profesional en distintas posiciones dentro de áreas de recursos humanos en distintas empresas. Además, se consideró su formación vinculada al área de recursos humanos.

La mayoría de las y los integrantes de esta muestra piloto poseían post grado y dobles carreras en su formación inicial. Para la aplicación de este piloto, se consideró un protocolo de contacto vía correo electrónico con las y los informantes, quienes contestaron durante los primeros días de septiembre de 2019 a través del sitio ORH Chile, Observatorio de Recursos Humanos ${ }^{15}$. Se envió una invitación on-line a la base de datos perteneciente al sitio especializado en contenido de gestión de personas con el link del instrumento publicado en el sitio mencionado, esto permitió contar con noventa y un (91) respuestas de representantes de empresas chilenas y multinacionales con presencia en el país. La plataforma digital especializada en encuesta que se utilizó para la recopilación y procesamiento de los datos fue SurveyMonkey ${ }^{16}$. La encuesta fue respondida entre la primera semana de septiembre y la última semana del mes de diciembre de 2019.

En cuanto a la muestra final con la que se trabajó fue del tipo intencional o por conveniencia (La Madriz, 2019) y estuvo constituida por 91 personas: 32 representantes de medianas y 51 informantes de grandes empresas de diversos rubros $^{17}$ de la Región Metropolitana de Chile registradas en

14 Especialmente en España, donde se han realizado en los últimos cuatro años estudios sobre el impacto de la transformación digital en las áreas de recursos humanos, lo que permitió definir las dimensiones del cuestionario vinculadas al objetivo del estudio y determinó la incorporación de la innovación como dimensión basal del proyecto.

15 Para más información acerca de este sitio web especializado ver en: https://www.observatoriorh.com/

16 Más información en https://es.surveymonkey.com/

17 Las y los informantes que integraron la muestra provenían de los siguientes rubros: retail (14), industria (10), inmobiliario y construcción (10), tecnología y telecomunicaciones (9), educación (8), energía (4), salud (4), banca (3), minería (3) y otros (26). 
la base de datos de ORH Chile y de la Red de Recursos Humanos ${ }^{18}$. La utilidad para el enfoque cuantitativo de una muestra de estas características es relativa y debemos señalar que los resultados que se obtuvieron son representativos solo para el grupo de organizaciones consultadas y no son extrapolables para grupos más amplios o estadísticamente representativos de la población (Hernández, Fernández y Baptista, 2014:171).

En la perspectiva de AvelloMartínez, et al, (2019), las tres principales limitantes del estudio fueron: 1) confidencialidad de los datos de las empresas: lo que implicó reducir al mínimo las preguntas que involucraban información privada ${ }^{19}$. 2) temática: los tópicos abordados en ciertos casos pudieron afectar contar con encuestas completas, en organizaciones que aún están en un nivel más administrativo u operativo y en donde los temas del estudio eran todavía muy incipientes. y 3) El haber trabajado con una muestra del tipo no probabilística, del tipo intencionada, dirigida o razonada no permite extrapolar los resultados obtenidos a grupos más amplios.

\section{Gestión de personas en empresas chilenas: hacia la innovación y transformación digital en su modelo de gestión}

Según el objetivo general de explicar de manera integrada las principales barreras que se presentan en las áreas de gestión de personas en empresas chilenas para incorporar la innovación y la transformación digital en su modelo de gestión, se exponen los principales resultados obtenidos de la aplicación de la encuesta. Se deja constancia que el orden de presentación no es un correlativo de las preguntas de la encuesta dado que aquí se hizo una selección para favorecer la síntesis en la exposición de los resultados.

Para vincular los resultados del estudio con el tipo de gestión de recursos humanos actualmente utilizado por las empresas participantes ${ }^{20}$, se solicitó como pregunta de entrada identificarse con la caracterización del modelo de los cuatro roles de Ulrich $(1997)^{21}$. En este caso, predominó el "experto en administración y gestión de funciones básicas", es decir en un ámbito más operativo con un $29 \%$. En un segundo lugar, con un $25 \%$, están los roles de "socio estratégico y agente de cambio". Señalar, que para estos roles su acción en la práctica se relaciona con la coordinación logística de actividades de apoyo a proyectos de transformación digital e innovación.

\subsection{Principales Resultados sobre Innovación}

Los siguientes tópicos consultados estuvieron orientados a identificar las principales barreras para innovar en las áreas de recursos humanos. Para

18 Un total aproximado de tres mil empresas (Universo de interés o de referencia).

19 Se solicitó información referida a tamaño y actividad y datos generales de identificación de la persona que respondía tales como: nombre, cargo, empresa y correo electrónico.

20 El 39\% correspondió a empresas medianas y el 61\% a empresas grandes. El detalle de los sectores está en nota al pie de página $\mathrm{N}^{\circ} 20$.

21 En esta pregunta respondieron 91 informantes y no hubo respuestas omitidas. 
Maliqueo Pérez, Carolina; González Candia, Julio; Mardones Espinosa, Regina; Ardiles Briones, Mauricio

Gestión de personas y las barreras para innovar en la transformación digital

estos efectos se preguntó sobre los procesos de cambio que ha liderado el área de Gestión de Personas (AGP), las acciones innovadoras, proyectos y metodologias desarrolladas, tabla 1.

Tabla 1
Procesos de cambios en el área de Gestión de Personas (AGP)
\begin{tabular}{llll}
\hline OPCIONES DE RESPUESTA & & RESPUESTAS \\
\hline Transformación Digital & $27 \%$ & 25 \\
\hline Ninguno & $27 \%$ & 25 \\
\hline Ambos, en forma independiente & $25 \%$ & 23 \\
\hline Innovación & $12 \%$ & 11 \\
\hline Otro (especifique) & $5 \%$ & 5 \\
\hline Ambos, porque son lo mismo & $2 \%$ & 2 \\
\hline TOTAL & & \\
\hline Respondidas: $\mathbf{9 1}$ - Omitidas: $\mathbf{0 . -}$ & & $\mathbf{9 1}$ \\
\hline Fuente: Maliqueo (2020)
\end{tabular}

Se destaca que un $27 \%$ de las y los encuestados señalaron la opción "ninguno". Con el mismo porcentaje, el proceso más mencionado fue el de "transformación digital". Por otra parte, las acciones innovadoras implementadas en el área son las siguientes, tabla 2:

Tabla 2

Acciones innovadoras AGP

\begin{tabular}{llll}
\hline OPCIONES DE RESPUESTA & & RESPUESTAS \\
\hline $\begin{array}{l}\text { Incorporación de algún tipo de tecnología digital para mejorar la } \\
\text { eficiencia de algunos procesos. }\end{array}$ & $68 \%$ & 52 \\
\hline $\begin{array}{l}\text { Nuevas formas de trabajar como equipo (trabajan por proyectos, } \\
\text { células) }\end{array}$ & $38 \%$ & 29 \\
\hline $\begin{array}{l}\text { Diseño de nuevos servicios para mejorar la experiencia del colabo- } \\
\text { rador }\end{array}$ & $26 \%$ & 18 \\
\hline $\begin{array}{l}\text { Creación de nuevos cargos para apoyar nuevos procesos o servi- } \\
\text { cios del área }\end{array}$ & $24 \%$ & 17 \\
\hline $\begin{array}{l}\text { Incorporación de nuevas profesiones al área de RRHH para impulsar } \\
\text { nuevas iniciativas }\end{array}$ & $22 \%$ & 14 \\
\hline $\begin{array}{l}\text { Diseño de una nueva estructura del área con nuevas unidades, por } \\
\text { ejemplo, unidad de Gestión del Cambio Innovación, etc. }\end{array}$ & $18 \%$ & \\
\hline
\end{tabular}




\section{Cont... Tabla 2}

\begin{tabular}{llr}
\hline Cambio en el organigrama, por ejemplo más horizontal & $17 \%$ & 13 \\
\hline Ninguna & $9 \%$ & 7 \\
\hline Total de encuestados: $\mathbf{7 6}$ & & \\
\hline Respondidas: $\mathbf{7 6}$ - Omitidas: 15.- & & \\
\hline
\end{tabular}

Fuente: Maliqueo (2020)

Las respuestas obtenidas a esta consulta dejan en evidencia la creencia común a nivel de conocimientos sobre la innovación en el sentido que sólo estaría relacionada con la implementación de tecnologías en los procesos (68\%). En segundo lugar, con un $38 \%$ de las preferencias se mencionaron nuevas modalidades o formas para trabajar en equipos, gráfico 1 .

De manera complementaria, se consultó sobre los proyectos nuevos que han incorporado en AGP durante los últimos 12 meses $^{22}$ :

\section{Gráfico 1 \\ Proyectos incorporados en la Gestión de Personas últimos 12 meses}

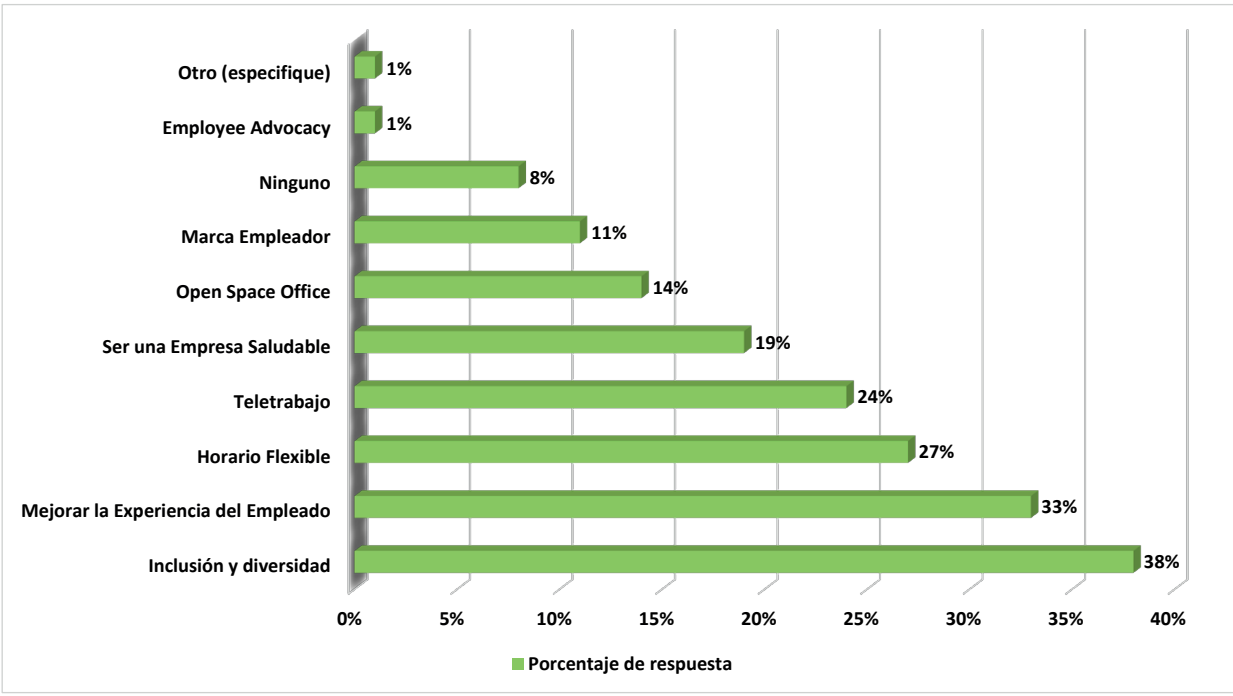

Fuente: Maliqueo (2020)

22 Respondidas: 76 - Omitidas: 15.- 
Maliqueo Pérez, Carolina; González Candia, Julio; Mardones Espinosa, Regina; Ardiles Briones, Mauricio

Gestión de personas y las barreras para innovar en la transformación digital

Los resultados se centraron en primer lugar en inclusión y diversidad (38\%), esta respuesta pudo estar condicionada a un aspecto legal dado que en el 2019 las empresas chilenas debían incorporarla en su gestión de recursos humanos ${ }^{23}$, lo que denota que los proyectos nuevos son parte de una reacción a las fuerzas del mercado y/o entorno, en este caso a la nueva legislación laboral. En la segunda posición aparece el concepto de experiencia del empleado que también responde a las tendencias que surgen en el momento y que son impulsadas por las consultoras.

Entendiendo que la innovación se relaciona también con la capacidad de resolver problemas creando soluciones efectivas, se indagó en el tipo de metodologías que utilizan las AGP, tabla 4:

Tabla 4

Metodologias AGP

\begin{tabular}{lrr}
\hline OPCIONES DE RESPUESTA & \multicolumn{2}{c}{ RESPUESTAS } \\
\hline Ninguna Metodología & $57 \%$ & 43 \\
\hline Agile & $14 \%$ & 11 \\
\hline Lean & $12 \%$ & 9 \\
\hline Design Thinking & $8 \%$ & 6 \\
\hline Mind Maps & $4 \%$ & 3 \\
\hline Scrum & $3 \%$ & 2 \\
\hline Canvas & $3 \%$ & 2 \\
\hline TOTAL & & 76 \\
\hline Respondidas: 76 - Omitidas: 15.- & & 2 \\
\hline
\end{tabular}

Fuente: Maliqueo (2020)

Los resultados evidencian un bajo uso de metodologías que son fundamentales para innovar o desarrollar proyectos en el área. Un $57 \%$ indica ninguna y sólo el $14 \%$ y $12 \%$ utilizan metodologías "Agile y Lean" respectivamente. Una metodología más o menos conocida es citada aquí con el $8 \%$ de las preferencias. Nos referimos a "design thinking". Para cerrar este primer eje de consultas, se preguntó sobre los principales obstáculos o barreras del AGP para Innovar en su modelo de gestión en la empresa ${ }^{24}$, gráfico 2 :

23 A contar del 1 de abril de 2019, las empresas que cuentan con 100 a 199 trabajadores deben cumplir con la Ley $\mathrm{N}^{\circ} 21.015$, que incentiva la inclusión laboral de personas con discapacidad, sumándose así a las empresas de 200 o más trabajadores, las que están obligadas al cumplimiento de esta ley desde el 1 de abril de 2018. En https://www.dt.gob.cl/portal/1626/w3-propertyvalue-167780.html visitada el 16 de marzo de 2021.

24 Se solicitó información referida a tamaño y actividad y datos generales de identificación de la persona que respondía tales como: nombre, cargo, empresa y correo electrónico. 


\section{Gráfico 2 \\ Barreras del área de Gestión de Personas para Innovar}

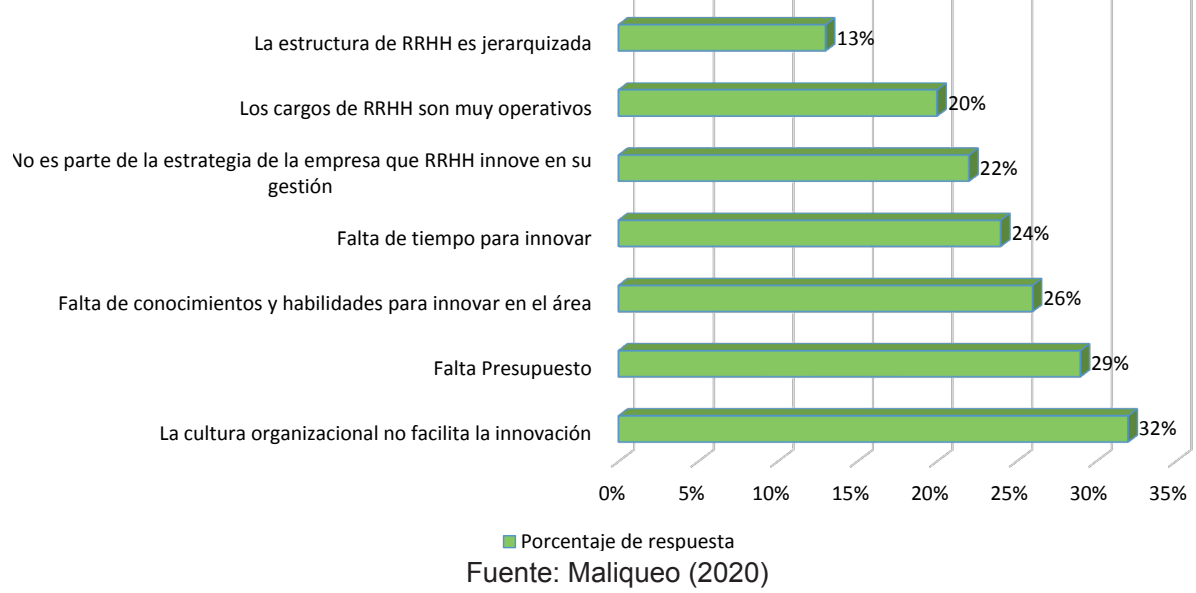

Las respuestas a esta pregunta demuestran que existen tres aspectos principales: cultura organizacional $32 \%$ que no facilita o que no es proclive a la innovación, la falta de presupuesto $29 \%$ y carecer de los conocimientos y habilidades necesarias para innovar con un $26 \%$ de las preferencias.

\subsection{Transformación Digital: principales resultados}

Un primer paso de indagación fue identificar si las áreas de recursos humanos estaban incorporando tecnología, para ello se consultó sobre si el AGP ha incorporado tecnología digital para mejorar su actual gestión ${ }^{25}$. Se destaca que el $82 \%$ de las empresas estudiadas han incorporado tecnologías. En este ámbito era necesario explorar en qué procesos se estaban implementando o habían incorporado algún tipo de tecnología digital, tabla 5 :

\footnotetext{
25 En esta pregunta respondieron 72 informantes y hubo 19 respuestas omitidas.
} 
Maliqueo Pérez, Carolina; González Candia, Julio; Mardones Espinosa, Regina; Ardiles Briones, Mauricio

Gestión de personas y las barreras para innovar en la transformación digital

Tabla 5

Procesos y Tecnologia Digital en AGP

\begin{tabular}{|c|c|c|}
\hline OPCIONES DE RESPUESTA & & RESPUESTAS \\
\hline Evaluación del desempeño & $49 \%$ & 35 \\
\hline Reclutamiento & $44 \%$ & 32 \\
\hline Remuneraciones & $42 \%$ & 30 \\
\hline Capacitación & $39 \%$ & 28 \\
\hline Comunicación Interna & $29 \%$ & 21 \\
\hline Selección & $28 \%$ & 20 \\
\hline Beneficios & $25 \%$ & 18 \\
\hline Contratación & $22 \%$ & 16 \\
\hline Clima & $22 \%$ & 16 \\
\hline Inducción & $18 \%$ & 13 \\
\hline Calidad de Vida & $13 \%$ & 9 \\
\hline Compensación total & $11 \%$ & 8 \\
\hline Sucesión y movilidad & $11 \%$ & 8 \\
\hline Ninguno & $10 \%$ & 7 \\
\hline Desvinculación & $6 \%$ & 4 \\
\hline Otro (especifique) & $1 \%$ & 1 \\
\hline \multicolumn{3}{|l|}{ Total de encuestados: 72} \\
\hline Respondidas: 72 - Omitidas: 19.- & & \\
\hline
\end{tabular}

Fuente: Maliqueo (2020)

Los resultados muestran que se han incorporado tecnologías para los procesos tradicionales como: evaluación de desempeño en un $49 \%$, reclutamiento con un $44 \%$ y remuneraciones con un $42 \%$. El tipo de tecnología utilizada responde a aquellas orientadas a la recopilación de datos y al uso de plataformas externas en procesos como reclutamiento, tabla 6 .

Para explorar en relación con el uso de tecnología más avanzada que se orientan más a un cambio de fondo o estructural en los modelos y a la transformación digital, se consultó sobre su uso en proyectos de recursos humanos. 
pp. 510-532

Revista Venezolana de Gerencia, Año 26 No. 94, 2021

\section{Tabla 6 \\ Proyectos Tecnologicos AGP}

\begin{tabular}{lll}
\hline OPCIONES DE RESPUESTA & & RESPUESTAS \\
\hline Modelo predictivos de sucesión & $7 \%$ & 5 \\
\hline Ranqueo de candidatos por inteligencia artificial & $1 \%$ & 1 \\
\hline Analíticos predictivos para cumplimiento formativo & $1 \%$ & 1 \\
\hline Inteligencia Artificial & $3 \%$ & 2 \\
\hline Machine learning para modelos predictivos de aprendizaje & $3 \%$ & 3 \\
\hline Machine learning & $3 \%$ & 3 \\
\hline Ninguno & $86 \%$ & 62 \\
\hline Otro (especifique) & $1 \%$ & 1
\end{tabular}

TOTAL: 72

Respondidas: 72 - Omitidas: 19.-

Fuente: Maliqueo (2020)

Se destaca que el $86 \%$ de las empresas no han incorporado este tipo de tecnologías más sofisticadas en sus procesos o proyectos. Este dato demuestra que están en una etapa inicial de digitalización lo que no significa que estén en un proceso de transformación digital. Para cerrar esta dimensión se consultó sobre las principales dificultades para incorporar tecnología en los procesos de Gestión de Personas ${ }^{26}$, gráfico 3.

26 En esta pregunta respondieron 72 informantes y hubo 19 respuestas omitidas. 


\section{Gráfico 3}

\section{Principales dificultades para incorporar tecnología en los procesos de Gestión de Personas}

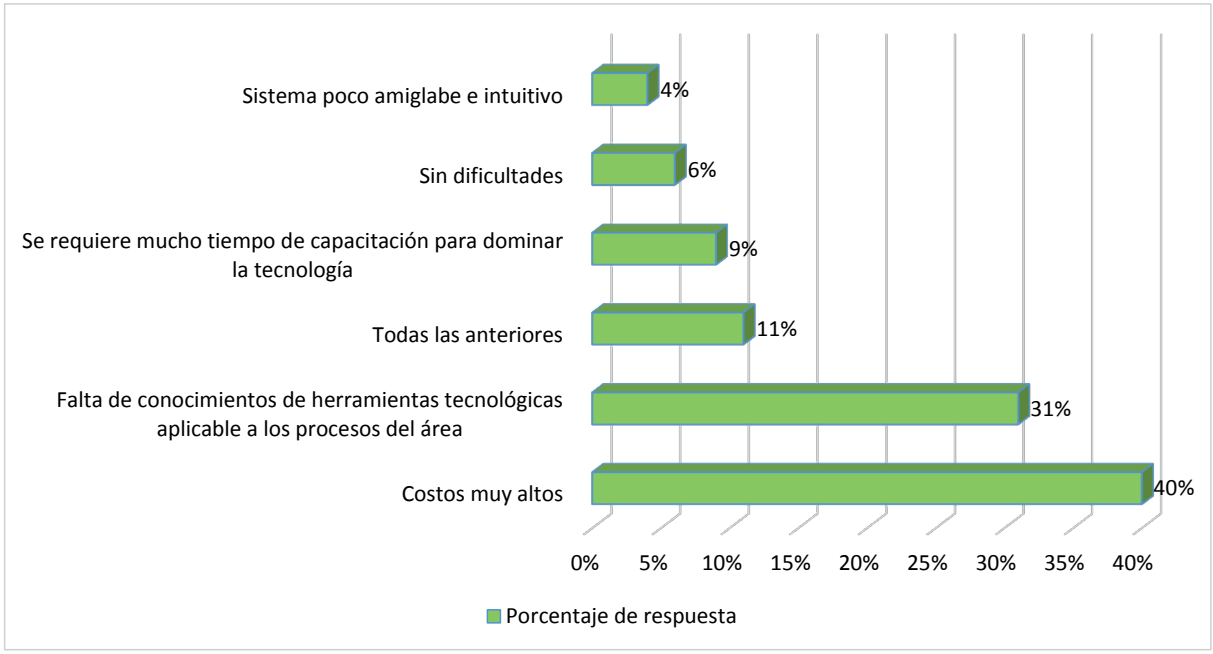

Fuente: Maliqueo (2020)

Los resultados indican que un $40 \%$ se relaciona con los altos costos y un $31 \%$ con la falta de conocimientos $y$ herramientas. Ambos porcentajes podrían estar vinculados puesto que dejan de manifiesto que la percepción de alto costo sea producto de la falta de conocimientos para comprender los beneficios que una implementación tecnológica podría aportar a la empresa y al área de recursos humanos para innovar, especialmente, en una perspectiva de mediano y largo plazo.

\subsection{Conocimientos: resultados}

El siguiente foco indagativo estuvo relacionado con el identificar si el grado de conocimiento, expresado en las capacitaciones realizadas, era un factor determinante en la actualidad para innovar en la transformación digital del AGP, tabla 7: 


\section{Tabla 7}

Capacitación en AGP

\begin{tabular}{lll}
\hline OPCIONES DE RESPUESTA & & RESPUESTAS \\
\hline Ninguno & $51 \%$ & 32 \\
\hline Metodología Agile & $27 \%$ & 17 \\
\hline Innovación & $21 \%$ & 13 \\
\hline Transformación Digital & $19 \%$ & 12 \\
\hline On-boarding & $14 \%$ & 9 \\
\hline Desing Thinking & $13 \%$ & 8 \\
\hline HR Analytics & $11 \%$ & 7 \\
\hline Experiencia del Empleado & $11 \%$ & 7 \\
\hline Creatividad & $10 \%$ & 6 \\
\hline Ciberseguridad & $10 \%$ & 6 \\
\hline $\begin{array}{l}\text { Habilidades Digitales (marketing digital, análisis de datos, gestión } \\
\text { de proyectos digitales) }\end{array}$ & $10 \%$ & 6 \\
\hline People Analytics & $8 \%$ & 3 \\
\hline Learning Machine/lnteligencia Digital & $5 \%$ & 1 \\
\hline Employee Advocacy & $2 \%$ & 1 \\
\hline Otro (especifique) & $2 \%$ & \\
\hline Total de encuestados: 63 & & \\
\hline Respondidas: 63 - Omitidas: 28.- & & \\
\hline Fuente: Maliqueo $(2020)$ & & \\
\hline
\end{tabular}

Fuente: Maliqueo (2020)

Los resultados obtenidos muestran que el $51 \%$ no han sido capacitados en algunas de las temáticas consultadas. Por otro lado, es interesante que se mencionaron las metodologías agiles y de innovación con un $27 \%$ y un $21 \%$ respectivamente. Lo anterior, nuevamente podría responder también a una tendencia o moda más que a una planificación estratégica o a una transformación en la forma de trabajo. Respecto de las o los integrantes del equipo que han sido capacitados, se obtuvo ${ }^{27}$, gráfico 4 : 
Maliqueo Pérez, Carolina; González Candia, Julio; Mardones Espinosa, Regina; Ardiles Briones, Mauricio

Gestión de personas y las barreras para innovar en la transformación digital

\section{Gráfico 4 \\ Colaboradores capacitados}

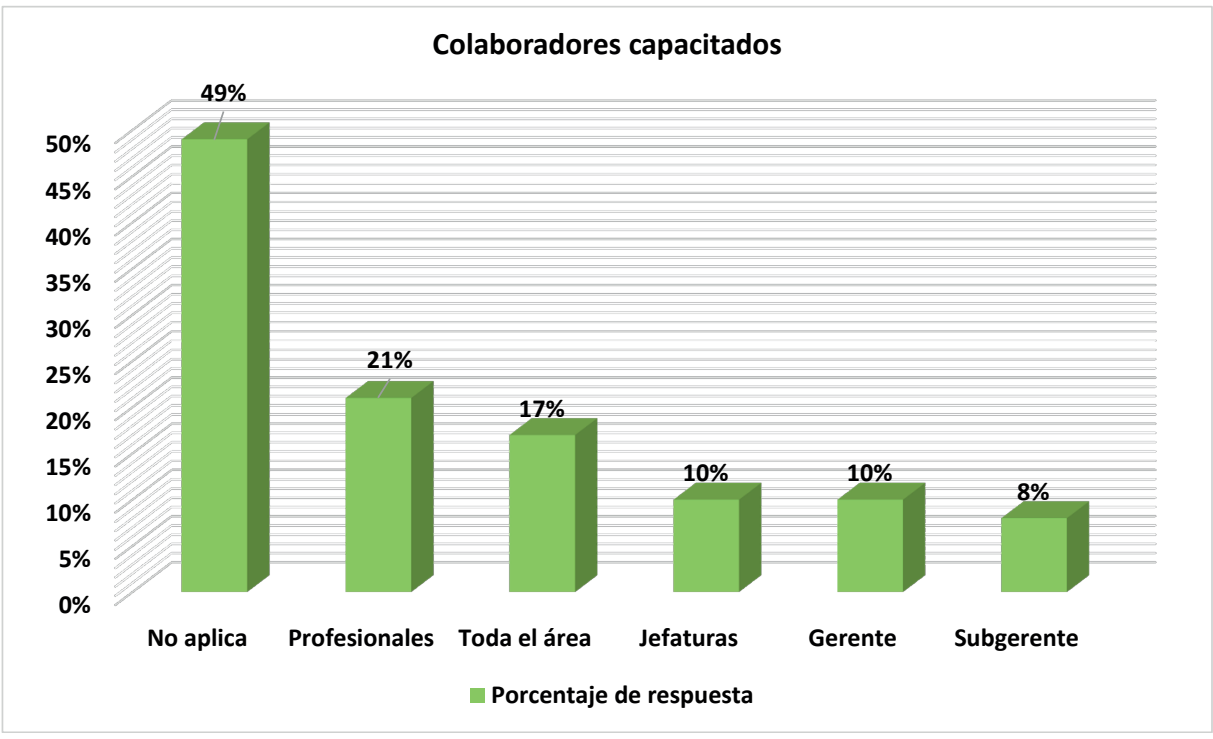

Fuente: Maliqueo (2020)

Las respuestas muestran datos interesantes con relación a los cargos de liderazgo, en el sentido que estos no han sido principalmente capacitados en estos temas. Nos referimos a los cargos de tipo gerencial y jefaturas. El principio general es que la o el líder del área impulsa el cambio a partir de decisiones estratégicas que responden a las demandas del cliente. En este estudio, al contrario de la recomendación general, se han capacitado preferentemente a los profesionales del equipo, quienes toman decisiones más bien operativas y tácticas.
Con relación a la planificación de los aprendizajes o la capacitación en estas materias ${ }^{28}$, se detectó que un $41 \%$ de las y los respondientes indican que son actividades aisladas realizadas interna o externamente y un $50 \%$ que no aplica o que no ha habido planificación. Solo un $10 \%$ señala que hubo presencia de un programa integral diseñado para el AGP. Lo anterior, nos evidencia que la falta de conocimientos planificados, estructurados y con los contenidos que respondan a decisiones estratégicas de formación en el área, se convierte en una de las principales barreras para innovar

28 En esta pregunta respondieron 63 informantes y hubo 28 respuestas omitidas. 
en la transformación digital. A modo de cierre de esta sección podemos señalar que las barreras que se presentan en las AGP en las empresas chilenas que participaron del estudio, para incorporar la innovación y la transformación digital en su modelo de gestión y transformarse en un agente de cambio para la organización, desde una perspectiva integrada fueron, diagrama 2 :

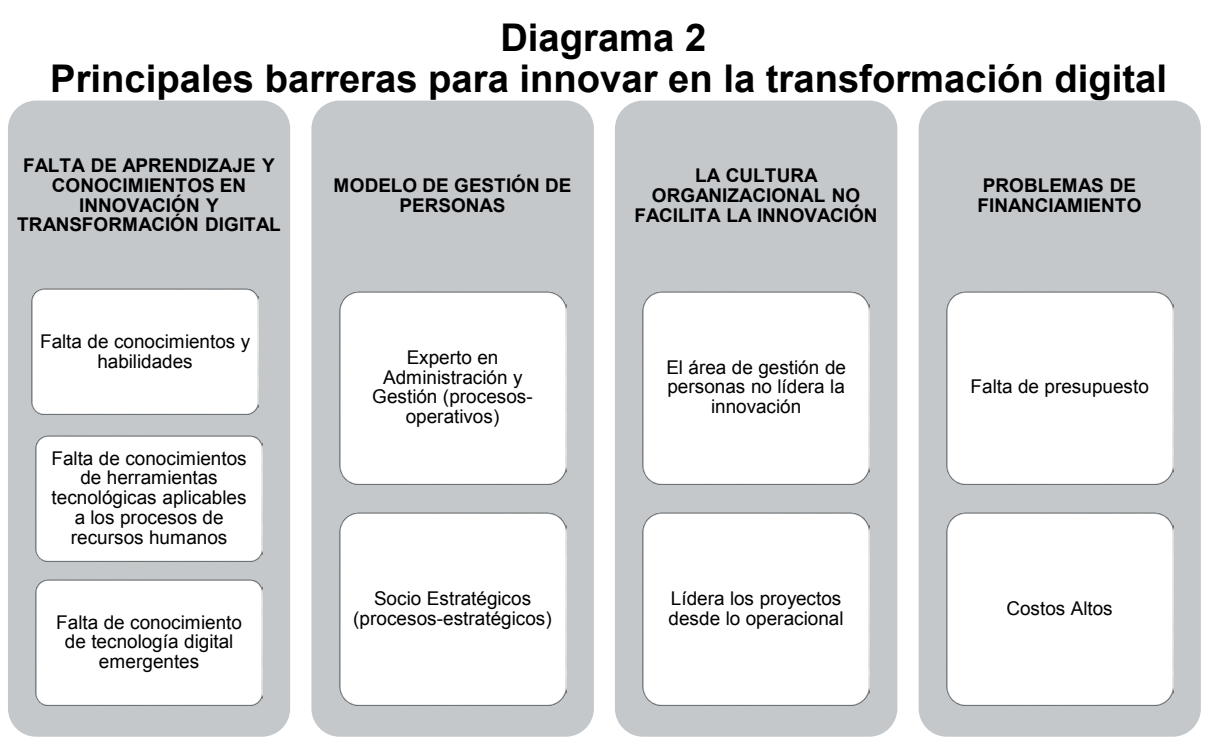

Fuente: Maliqueo (2020)

\section{Conclusiones}

En este estudio quedó en evidencia que las AGP están en una etapa de digitalización de sus procesos utilizando tecnologías tradicionales como nuevas versiones de los softwares que utilizan para la evaluación del desempeño, administración de las remuneraciones y beneficios, es decir, en procesos operativos básicos del área. Aún están muy distantes del uso de tecnologías emergentes o avanzadas como la inteligencia artificial. La mayoría de las y los representantes de las empresas consultadas se identifican como expertos en administración y de procesos, lo que refleja el uso de tecnología más tradicional en sus operaciones.

Para poder innovar en la transformación digital del AGP es fundamental que las y los profesionales sean capacitados para abordar estos desafíos, tanto en temas conceptuales referentes a innovación, tipo de tecnologías, herramientas tecnológicas y 
Maliqueo Pérez, Carolina; González Candia, Julio; Mardones Espinosa, Regina; Ardiles Briones, Mauricio

Gestión de personas y las barreras para innovar en la transformación digital

transformación digital, y sin lugar a duda, en el diseño, planificación, evaluación e implementación de proyectos de este tipo. Por otro lado, se requiere capacitar en las competencias conductuales y habilidades socioemocionales, que sostengan los cambios culturales que supone la transformación digital, tales como: la capacidad de resolver problemas complejos, pensamiento crítico, creatividad, colaboración con otros, toma de decisiones asertivas, orientación al servicio, negociación y desarrollo de relaciones positivas. Además de tecnologías avanzadas aplicables al AGP, como inteligencia artificial, big data y de metodologías de análisis como HR analytics, entre otros.

Las empresas que participaron en este estudio señalan, en un $29 \%$, que en su actual rol se autodefinen en un nivel de experto en administración en procesos operativos, es decir, la mayoría están realizando funciones de orden básico de acuerdo con el modelo de Ulrich (1997). En un segundo lugar, con un $25 \%$, emerge el rol de socio estratégico, es decir, vinculando los procesos operativos a los lineamientos de más largo plazo e importancia relativa entregados por la organización.

Dado que los roles predominantes en las grandes empresas se vinculan con procesos más bien de nivel operativo, la participación en proyectos de nivel estratégico se limita a dar apoyo a nivel de coordinación, planificación y logística, de los requerimientos que surgen de la estrategia. Los resultados del estudio indican que el $27 \%$ del AGP no lideran ningún proceso de cambio y sólo un $12 \%$ lidera un programa de innovación dentro de las empresas. Vinculado con este último dato un $57 \%$ de las y los consultados declaran no aplicar ninguna metodología para resolver las problemáticas de la organización.

Finalmente, y en un contexto de intensa transformación digital empresarial, donde la experiencia y capacitación de las y los trabajadores, su desarrollo y fidelización son requisitos imprescindibles para la viabilidad futura de las organizaciones, se necesita un re-enfoque estratégico de la visión de la gerencia general hacia la gestión de personas y un cambio de la función del área, de sus procesos y servicios, un nuevo modelo de gestión de personas que sea liderado efectivamente por sus directivos y que utilice la innovación y la digitalización como factores críticos de éxito para la transformación digital, que es e implica también una profunda transformación cultural.

\section{Referencias Bibliográficas}

Alexander, T., Stefanova, V., y Zahidi, S. (2018). The Future of Jobs Report 2018. Cologny/ Geneva (Switzerland): World Economic Forum. http://www3.weforum.org/ docs/WEF Future of Jobs 2018. pdf

Altimiras, M. (2017). Cómo los recursos humanos pueden manejar los retos de la transformación digital. https:// bit.ly/32E $47 \mathrm{bL}$

Avello-Martínez, $\quad$ R., $\quad$ RodríguezMonteagudo, M., RodríguezMonteagudo, P., Sosa-López D, Companioni-Turiño, B. RodríguezCubela, R. (2019) ¿Por qué enunciar las limitaciones del estudio? Medisur, 17(1). http://www.medisur.sld.cul index.php/medisur/article/view/4126

Baena, G. (2017). Metodología de la Investigación. Serie Integral por competencias. Tercera edición Ebook. https://bit.ly/3xgIRXJ

Barros, P. (2016). Transformación digital: 
¿Cómo está cambiando la forma en la que trabajamos? Revista $\mathrm{ORH}$, 116, 12.

Cobo, J. (2008). El concepto de tecnologías de la información. Benchmarking sobre las definiciones de las TIC en la sociedad del conocimiento. Zer, 14(27), 295-318. https://bit.ly/3tOGp8U

Crespo, B. y Pariente, E. (2018). Barómetro sobre la madurez digital en España 2018. Madrid: Divisadero. A Merkle Company - IE Business School. https://www.divisadero.es/ wp-content/uploads/barometromadurez-digital-espana-2018.pdf

Cuenca-Fontbona, J., Matilla, K. y Compte-Pujol, M. (2020). Transformación digital de los departamentos de relaciones públicas y comunicación de una muestra de empresas españolas. Revista de Comunicación, 19(1), 2227-1465. https://revistadecomunicacion.com/ article/view/1640/1516

Décima Encuesta de Innovación en Empresas, 2015-2016, Ministerio de Economía, Fomento y Turismo. https://www.economia.gob. cl/2018/02/22/decima-encuesta-deinnovacion-en-empresas-2015-2016. $\underline{\mathrm{htm}}$

Denning, S. (2016). Cómo hacer que toda la organización sea ágil. Estrategia y liderazgo, 44(4), 10-17. https://doi. org/10.1108/SL-06-2016-0043

Eguren, Á. (2019). La gestión de recursos humanos como elemento clave en el desarrollo de programas de emprendimiento corporativo. Trabajo fin de Grado en Relaciones Laborales Curso Académico 20182019. Universidad de Cantabria. https://bit.ly/3vgEAlp

Fitzgerald, M., Kruschwitz, N., Bonnet, D., y Welch, M. (2013). Embracing
Digital Technology a New Strategic Imperative. Mit Sloan Management Review. https://emergenceweb.com/ blog/wp-content/uploads/2013/10/ embracing-digital-technology.pdf

Hernández-Samiperi, R., Collado, C. y Baptista, M. (2014). Metodología de la Investigación. Sexta Edición. Editorial Mc Graw Hill.

Hernández-Sampieri, R. \& Mendoza, C (2018). Metodología de la investigación. Las rutas cuantitativa, cualitativa y mixta. Editorial Mc Graw Hill Education.

Herrera, R., y Hidalgo, A. (2019). Dinámica de la gestión de la innovación de servicios y co-creación en empresas del sector economía digital. Contad. Adm. 64(1). https:// bit.ly/3gC8FaH

Jiménez, A., y Geldes, C. (2019). Los desafíos de la innovación en Latinoamérica. Journal of Technology Management \& Innovation, 14(4). https://bit.ly/3dEE6iX

Khin, S., y Ho, TC (2019). Tecnología digital, capacidad digital y desempeño organizacional: un papel mediador de la innovación digital. Revista Internacional de Ciencia de la Innovación, 11(2), 177-195. https:// doi.org/10.1108/IJIS-08-2018-0083

La Madriz, J. (2019). Metodología de la Investigación. Actuación Humana orientada al conocimiento de la realidad observable. CIDE. Centro de Investigación y Desarrollo Ecuador. https://bit.ly/2QQ0KvS

Maliqueo, C. (2019). Dimensiona la transformación digital en su justa medida. Revista Digital ORH https:// observatoriorh.cl/dimensiona-latransformacion-digital-en-su-justamedida/

Maliqueo, C. (2020). Barreras del 
Maliqueo Pérez, Carolina; González Candia, Julio; Mardones Espinosa, Regina; Ardiles Briones, Mauricio

Gestión de personas y las barreras para innovar en la transformación digital

área de gestión de personas para innovar en la transformación digital: estudio realizado en 91 empresas de la región metropolitana de Chile. https://bit.ly/3elkwYg

Maliqueo, C. y González, J. (2020). Diseño y validación de un instrumento para medir el nivel de madurez en innovación y transformación digital en la gestión de personas. E-Journal Técnica Administrativa, 19(3). http://www.cyta. com.ar/ta/article. php? id=190306

Martínez, N. y Bello, M. (2017). Claves para conseguir una transformación digital de verdad, observatorio de Recursos Humanos. Revista $\mathrm{ORH}$, 118,13

Mckinsey, (2019). So funktioniert die Transformation. https://bit.ly/3ehqLvS

Ministerio de Ciencia, Tecnología, Conocimiento e Innovación. Gobierno de Chile (2020). Encuesta Nacional de Innovación y Encuesta Nacional de I + D. https://bit.ly/3tR7jg1

OECD/Eurostat (2018). El Manual de Oslo, 20-21.

Piña, L. E., \& Senior, A. (2020). Estudio de la ciencia, tecnología e innovación desde perspectivas multitécnicas. Revista de Ciencias Sociales, 26(3), 312-326. https://doi. org/10.31876/rcs.v26i3.33251

Power Data. (2020). Transformación digital. Qué es y su importancia y relación con los datos. https://www. powerdata.es/transformacion-digital

Reyes, N., y Boente, A. (2019). Metodología de la Investigación Compilación Total. https://bit. Iy/3gBaRkX
Schwab (2016) Strategic Intelligence. https://bit.ly/3xj6Mpn

Torrecilla, J., Pardo, C., y Rubio, J. (2019) Industria 4.0 y transformación digital. Revista de trabajo y seguridad social. Estudios financieros. Revista de trabajo y seguridad social: Comentarios, casos prácticos: recursos humanos, N. Extra 1, 2754. https://bit.ly/3eiWuwU

Torres, L. C., y Vargas, G. G. (2020). Complejidad y la organización. Revista Electrónica Gestión de las Personas y Tecnologías, 13(37). https://www. revistagpt.usach.cl/sites/revistagpt/ files/complejidad y la organizacion. pdf

Ulrich, D. (1997). Recursos Humanos Champions. Buenos Aires, Argentina: Granica.

Ulrich, D. (2006). La propuesta de valor de Recursos Humanos. Editorial Deusto, Barcelona

Uzkudun, I. (2014). Cómo puede RRHH contribuir más al negocio: Dave Ulrich y los 4 roles de Recursos Humanos. https://bit.ly/2QwUb1d

Vilaplana, F., y Stein, G. (2020). Digitalización y personas. Revista Empresa y Humanismo, XXIII(1),113. https://revistas.unav.edu/index. php/empresa-y-humanismo/article/ view/38562/33944

Walker, M., Blosch, M., Burke, B., Burton, B., Brand, S., y Weldon, L. (2016). Enterprise Architecture and Technology Innovation Leadership Vision 2017. Gartner, https://gtnr. it/3nlv2mr 